\title{
PERCEPCIONES Y HÁBITOS DE CONSUMO DE LA LECHE DE CABRA Y SUS DERIVADOS EN LOS COSTARRICENSES ${ }^{1}$
}

\author{
Alejandro Chacón-Villalobos ${ }^{2}$, Yorleny María Araya-Quesada ${ }^{3}$, Marta Eugenia Gamboa-Acuña ${ }^{3}$
}

\begin{abstract}
RESUMEN
Percepciones y hábitos de consumo de la leche de cabra y sus derivados en los costarricenses. En el segundo semestre de 2006 se efectúo un estudio básico de consumidor empleando una metodología de encuesta aleatoria con 507 costarricenses distribuidos en todo el país según proporción demográfica (69\% mujeres y $31 \%$ hombres entre 12 y 70 años); se consideró la edad, género, ubicación geográfica, práctica de ejercicio, percepciones hacia la leche de cabra y sus derivados, así como hábitos y frecuencia de consumo. Se pretendió determinar interrelaciones entre las variables de estudio. Los datos se evaluaron con pruebas de chi cuadrado. La población se caracterizó por una tendencia marcada hacia el ejercicio en el sexo masculino no asociada a la edad de las personas, y que no influye en el consumo de leche caprina. Las principales percepciones hacia esta leche fueron el ser "saludable" (36\%), "nutritiva" (14\%) y "medicinal" (14\%). Un $93,7 \%$ de los entrevistados no consumen leche de cabra principalmente debido a la escasa disponibilidad (31,2\%), la sensación de náusea $(31,2 \%)$ y el desconocimiento hacia el producto $(14,6 \%)$. El 6,31 \% de los encuestados consumen la leche entre una y dos veces por semana $(53,1 \%)$, principalmente grupos de mayor edad radicados en San José y Cartago, dónde compran directamente a los productores $(68,75 \%)$ y motivados por su salud (75\%). Un 11,24\%, principalmente radicado en el Valle Central, consume derivados, principalmente queso $(80 \%)$ y yogurt $(10 \%)$. Estos derivados son adquiridos con una frecuencia variable por medio de compra a pequeños detallistas $(38,6 \%)$, o directamente con el productor $(36,8 \%)$. La creencia en la leche caprina como un nutracéutico no se traduce en un mayor consumo.
\end{abstract}

Palabras clave: Preferencia, lácteos caprinos, hábitos nutricionales, conocimiento, opinión.

\begin{abstract}
Perceptions and consumption habits of Costa Ricans toward goat's milk and dairy. A study of consumer habits and perceptions related with goat milk and goat dairy took place among 507 Costa Ricans randomly distributed by demographics in the country (69\% females, $31 \%$ males aged between 12 and 70 years), during the second semester of 2006. Random interviews were used to profile age, gender, location, exercise practices, consumption habits, perceptions and knowledge toward goat milk and dairy. Coded data were evaluated using the chi square test. A tendency for significantly more exercise among men was found, which was not related to age or milk consumption. The perceptions of goat milk as "healthy" (36\%), "nutritious" (14\%) and "medicinal" (14\%) where cited the most. About $93.7 \%$ of the persons mentioned goat milk as being "not available" $(31.3 \%)$. "Repulsion for the milk itself" $(31.2 \%)$ and "lack of knowledge" (14.6\%) were the main reasons for not consuming goat milk. $6.31 \%$ did consume goat milk once or twice a week $(53.1 \%)$, especially older people in San José and Cartago, who bought milk directly to the farmers $(68.75 \%)$ and were motivated by health benefits (75\%). About $11.24 \%$ of the persons, especially those located at the Costa Rican central plateau, usually consumes goat dairy, mainly cheese (80\%) and yogurt (10\%). Dairy is bought with variable frequencies in small rural convenience stores (38.6\%) and directly to the farmers $(36.8 \%)$. Although most of the people thinks of goat milk as a nutraceutic, this idea does not translate into a high consumption of the product.
\end{abstract}

Key words: Liking, goat dairy, nutritional habits, knowledge, opinion.

\footnotetext{
1 Recibido: 28 de noviembre, 2007. Aceptado: 26 de agosto, 2008. Proyecto inscrito en Vicerrectoría de Investigación No.737-A4-040, Universidad de Costa Rica.

2 Estación Experimental Alfredo Volio Mata, Facultad de Ciencias Agroalimentarias. Universidad de Costa Rica. San José, Costa Rica. alejandro.chacon@ucr.ac.cr

3 Escuela de Tecnología de Alimentos, Facultad de Ciencias Agroalimentarias. Universidad de Costa Rica. San José, Costa Rica. yorleny. araya@ucr.ac.cr; egamboa@clemson.edu
} 


\section{INTRODUCCIÓN}

La industrialización de los productos de la leche constituye uno de los sectores agroindustriales más representativos de la economía costarricense (Díaz 2004). Así mismo, la ingesta de este tipo de productos alimenticios crece constantemente entre la población del país (Vega 2002). El consumo de lácteos en Costa Rica es de hecho uno de los más altos de América Latina (Morón et al. 2005). Factores demográficos y socioculturales tales como la edad, el género, el estilo de vida, el nivel socio económico y académico, así como la disponibilidad del producto, han mostrado ser de alta influencia como determinantes del consumo en este mercado particular (Ivankovich et al. 1990, Severi y Girona 2005, Carriquiry 2005, Vargas et al. 2007).

A pesar del dinamismo y diversificación de productos que caracteriza al sector lácteo costarricense, el mismo se basa de manera casi exclusiva en derivados de la leche de vaca (Corrales y Chacón 2005, Rojas et al. 2007, Vargas et al. 2007). Prejuicios de índole cultural, y la poca empatía sensorial, han causado que otras fuentes lácteas alternativas como la leche de cabra o de búfala no cuenten con una amplia comercialización (Corrales y Chacón 2005). Este tipo de lácteos no tradicionales en el país se producen por lo tanto a una escala muy artesanal, siendo muy limitadas sus cadenas de distribución y venta (Chacón 2004). Es una realidad que la leche de cabra y sus derivados representan un nicho comercial muy restringido, siendo la leche fluida fresca la más comercializada, y a un precio de venta mayor que la leche vacuna, pues la cultura popular le atribuye propiedades nutracéuticas que hacen que ciertas personas la compren como remedio de manera muy ocasional (Tacsan 1987).

Para la leche caprina en particular, son muy escasos los estudios que han evaluado las percepciones y hábitos de los consumidores en el mercado costarricense, los cuales podrían explicar en parte el panorama existente. En un estudio de Vargas et al. (2007) efectuado entre estudiantes universitarios, hasta un $40 \%$ de la población evaluada ignoraba por completo detalle alguno sobre la leche de cabra o respecto a los lácteos caprinos, no habiendo probado nunca en su vida ningún derivado un total de 33,5\% de los educandos. Corrales y Chacón (2005), encontraron en una muestra experimental que $54 \%$ de los costarricenses encuestados no habían probado ni al menos una vez en sus vidas queso de cabra de ningún tipo.

La leche de cabra, por sus múltiples propiedades nutracéuticas (Chacón 2005); por el alto rendimiento de sus productos derivados (Corrales y Chacón 2005), y dadas las características de alta eficiencia y poca demanda de las cabras como animal lechero (University of Maryland 1992), representa una alternativa comercial interesante en la actualidad, por lo cual evaluar la percepción que la misma tiene entre el consumidor costarricense es de primordial interés. Tal y como sugirieron Severi y Girona (2005), este tipo de evaluaciones ampliaría la comprensión de los hábitos de consumo permitiendo el desarrollo y adopción de estrategias de promoción adecuadas. Se puede así descubrir las necesidades y deseos de grupos o segmentos de mercado específicos, orientando la promoción y mercadeo hacia sus necesidades particulares (Schiffman y Lazar 2001). Una manera efectiva para determinar la percepción de los consumidores sobre la leche de cabra y sus derivados, así como sus hábitos, es a través de la encuesta, la que ha mostrado ser útil en estudios anteriores (Orozco 1999).

El trabajo que se presenta tuvo como objetivo principal estudiar entre la población costarricense las percepciones y hábitos de consumo de la leche de cabra y sus derivados, estudiando las relaciones existentes con el estilo y condición de vida.

\section{MATERIALES Y MÉTODOS}

El estudio se efectuó entre los meses de junio y diciembre del año 2006 en las siete provincias de la República de Costa Rica. La tabulación y análisis de la información se efectuó en la Estación Experimental de Ganado Lechero Alfredo Volio Mata (ubicada en Ochomogo, Cartago) y en la Escuela de Tecnología de Alimentos (localizada en San Pedro de Montes de Oca, San José). Ambas unidades académicas pertenecen a la Facultad de Ciencias Agroalimentarias de la Universidad de Costa Rica.

El trabajo consistió en una evaluación de percepciones y hábitos de consumo de leche y lácteos caprinos en 507 personas de nacionalidad costarricense 
(69\% mujeres y $31 \%$ hombres), con un rango de edades comprendido entre los 12 y 70 años. La población muestral a evaluar se estratificó proporcionalmente en las siete provincias del territorio costarricense, en base a los datos demográficos del Instituto Nacional de Estadística y Censos (2005), tal y como lo muestra el Cuadro 1.

Cuadro 1. Distribución porcentual de la población muestral según provincia de residencia. San José, Costa Rica. 2007.

\begin{tabular}{lc}
\hline Provincia & Porcentaje de la población muestral $(\boldsymbol{\%})$ \\
\hline San José & 36,3 \\
Alajuela & 17,6 \\
Cartago & 11,0 \\
Limón & 9,7 \\
Heredia & 9,3 \\
Puntarenas & 9,5 \\
Guanacaste & 6,7 \\
\hline
\end{tabular}

Con la finalidad de recopilar la información necesaria, se diseñó un formulario de encuesta según las recomendaciones de Corrales y Chacón (2005), Rojas (2005) y de Ivankovich (2003) ${ }^{5}$. Esta herramienta incluyó preguntas cerradas para caracterizar la población en cuanto a edad, género, estado civil, nivel académico, práctica de ejercicio físico y hábitos de consumo de lácteos caprinos. Se incluyeron además preguntas abiertas sobre el conocimiento y opinión general hacia los lácteos caprinos.

La encuesta fue aplicada en forma aleatoria a la población de cada una de las provincias hasta completar el tamaño muestral, empleando para ello la metodología de entrevista.

Los datos recopilados fueron codificados para cada ítem de la encuesta, tabulando posteriormente dicha información en cuadros de frecuencia para su evaluación estadística. Empleando el programa estadístico JMP-SAS versión 4.0 se efectuaron pruebas

\footnotetext{
5 Ivankovich, C. 2003. Sondeo de mercado con consumidores habituales. Centro Nacional de Ciencia y Tecnología de Alimentos. San José. Costa Rica. Comunicación personal.
}

de chi cuadrado $\left(\mathrm{X}^{2}\right)$ (5\% de significancia) para valorar la relación entre las variables categóricas edad, género, ubicación geográfica, práctica de ejercicio, y conocimiento y percepción sobre la leche de cabra. Se establecieron los principales efectos que explicaron las relaciones encontradas, con el empleo de gráficas y cuadros de contingencia generados por el programa informático, tal y como recomienda O’Mahony (1986).

\section{RESULTADOS Y DISCUSIÓN}

La distribución de la población evaluada según edad se muestra en el Cuadro 2.

Cuadro 2. Distribución porcentual de los entrevistados sobre la percepción y hábitos de consumo de leche y lácteos caprinos, según grupo de edad. San José, Costa Rica. 2007.

\begin{tabular}{ccc}
\hline Edades & Grupo de edad & Porcentaje (\%) \\
\hline 12 a 19 & Adolescentes & 11,6 \\
20 a 29 & Adulto joven & 26,6 \\
30 a 49 & Adulto & 33,3 \\
Más de 50 & Adulto mayor & 28,4 \\
\hline
\end{tabular}

La muestra, aunque fue aleatoria, guardó cierta simetría entre grupos de edad en cuanto al porcentaje de personas entrevistadas, siendo el caso de los adolescentes quizás el minoritario como se observa en el Cuadro 2. Esta particularidad puede estar relacionada con la poca apertura de los adolescentes a contestar cuestionarios, así como porque al ser efectuada la entrevista en horas hábiles, muchas personas pertenecientes a este grupo de edad se encontraban en sus respectivos lugares de estudio. Idénticas razones, asociadas a la disponibilidad horaria, podrían explicar el hecho de que fueran más las mujeres amas de casa participantes que los hombres.

Dadas las propiedades nutracéuticas de la leche de cabra, se consideró de interés estudiar si el consumo de la misma se asoció con hábitos de vida saludable como el ejercicio, aspecto considerado como relevante en otros estudios similares efectuados en el país (Vargas et al. 2007). 


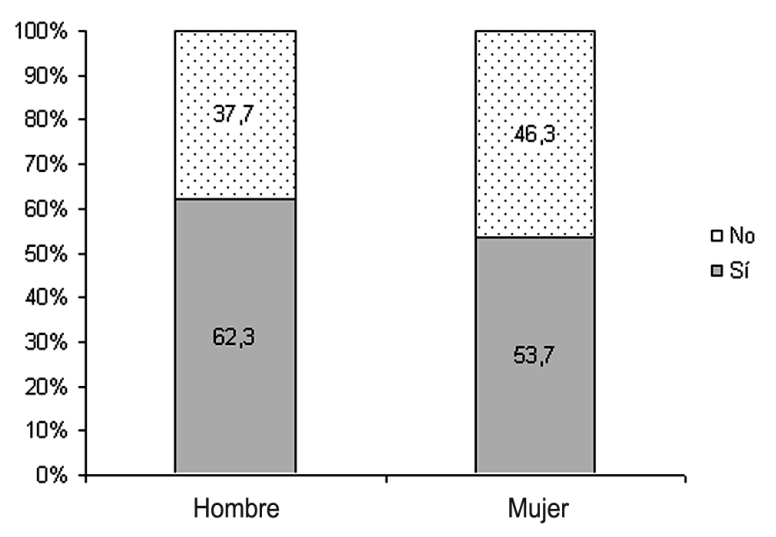

Figura 1. Distribución del ejercicio físico según género entre la población muestral encuestada sobre la percepción y hábitos de consumo de leche y lácteos caprinos. San José, Costa Rica. 2007.

Con la intención de caracterizar a la población encuestada, se efectuó un análisis logístico que encontró la existencia de una relación $\left(X^{2}=3,252 ; p=0,0713\right)$ entre la práctica de algún tipo de ejercicio físico y el género de las personas. Con la intensión de evaluar el patrón del comportamiento de la distribución, se inspeccionaron los datos gráficamente para éste y todos los casos, según recomienda O’Mahony (1986). Tal y como lo muestra la Figura 1, el comportamiento de los datos muestra que el hábito de realizar ejercicios es notablemente más marcado entre hombres que en el sexo femenino.

Los resultados obtenidos concuerdan con aquellos reportados en el país por Vargas et al. (2007) para estudiantes universitarios costarricenses. En dicho estudio se lograron detectar relaciones significativas entre la práctica de ejercicio físico según el género, siendo igualmente la proporción de hombres que se ejercitan mayor que la de mujeres. Así mismo, los datos guardan ciertos paralelismos con los reportados por Chacón y Moncada (2005), quienes encontraron que en una población muestral de costarricenses, un $60 \%$ de las personas en promedio realizaban algún tipo de actividad física, en contraposición con un $36 \%$ que no participaba en actividades de este tipo. Encuestas como la realizada por el Ministerio de Salud de Costa Rica (2001), igualmente reflejan similares porcentajes de actividad física, cercanos al 63,2\%.

Para complementar la información anterior se estudió la relación entre la práctica del ejercicio y

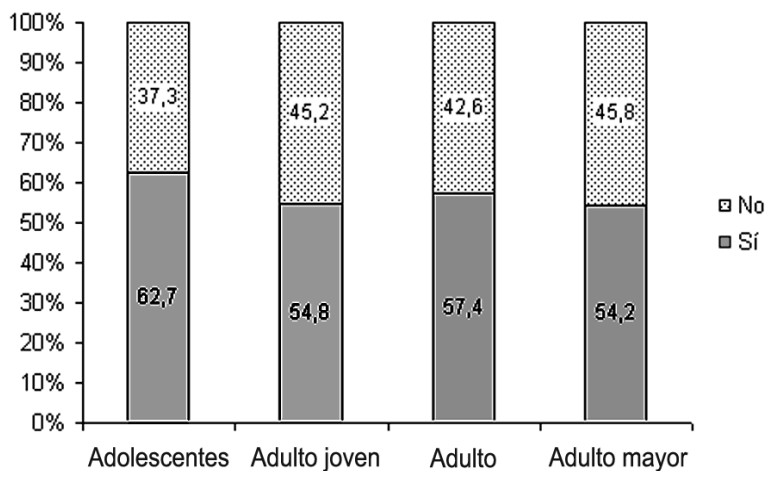

Figura 2. Distribución del ejercicio físico según grupo de edad, con base en la encuesta sobre la percepción y hábitos de consumo de leche y lácteos caprinos. San José, Costa Rica. 2007.

la edad, encontrándose por medio de una prueba de chi cuadrado que no existió una relación entre los diferentes grupos $\left(X^{2}=1,466 ; p=0,6901\right)$. Los porcentajes correspondientes se muestran en la Figura 2.

Los datos obtenidos en conjunto, así como los reportados en la bibliografía, parecieran sugerir que el aparente sedentarismo femenino es mayor en la población costarricense aún indiferentemente al grupo edad al que pertenezca la persona, razón por la cual esta evidencia da pie a posibles estudios posteriores más detallados en este aspecto particular.

La población encuestada fue abordada en cuanto al tópico de cuál es su conocimiento principal o más relevante sobre la leche caprina y sus características. Ante esta interrogante el $36 \%$ de los participantes señaló que la leche de cabra es un producto que se caracteriza por ser saludable. Conceptos tales como que esta leche es "nutritiva" (14\%) y "medicinal" (14\%) destacaron entre las características de mayor mención por la población. Adicionalmente, un $22 \%$ de los encuestados presentaron una amplia diversidad de respuestas misceláneas agrupadas en la categoría de "otros", dónde se destacan observaciones como que la leche caprina "es mejor que la leche de vaca" $(5,3 \%)$; que "esta leche es apta para ser consumida por seres humanos" $(5,7 \%)$ y que "es buena para la salud de los niños" $(2,6 \%)$. Un punto de controversia parece surgir en torno al tópico del sabor, pues mientras un 2,2\% de la población abordada opina que esta leche posee un buen sabor, otro $1,4 \%$ sostiene lo contrario. 
Los resultados expuestos se resumen en la Figura 3 .

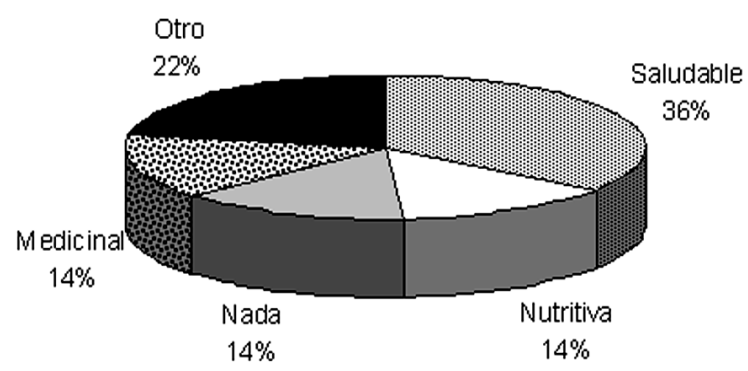

Figura 3. Percepción porcentual general de la población costarricense encuestada sobre la leche de cabra. San José, Costa Rica. 2007.

Partiendo de la información antes detallada, es interesante notar como la gran mayoría de las opiniones sobre la leche de cabra son positivas en términos de la concepción del producto como un alimento nutraceútico. Según Chacón (2005), las creencias manifestadas por los evaluados en este estudio, están de hecho respaldadas por el conocimiento científico actual, por lo cual el conocimiento popular no se encuentra sesgado en este particular. Esta tendencia de las opiniones hacia el ámbito positivo en cuanto a la percepción de la leche caprina como un alimento saludable, concuerdan con lo evidenciado por Vargas et al. (2007) entre estudiantes universitarios. Lo anterior es extrapolable a productos como el queso caprino, el cual según lo expuesto por Corrales y Chacón (2005), es considerado como nutritivo por un $35,6 \%$ de los costarricenses encuestados $(\mathrm{n}=59)$, y como saludable por un $27,1 \%$. La percepción nutricionalmente positiva reflejada por la suma de los dos apartados anteriores sería entonces de un $62,7 \%$.

Con la intención de estudiar si existe una relación entre el hábito del ejercicio y el conocimiento sobre la leche de cabra, quizás asociados ambos factores dentro de un esquema de vida saludable a la luz de las opiniones recopiladas, se procedió a efectuar un análisis logístico para ambos aspectos. Se encontró para la población estudiada que no existió una relación $\left(X^{2}=2,794 ; p=0,5928\right)$ entre los conocimientos sobre la leche de cabra y la realización de ejercicios. No es posible afirmar que las personas que hacen ejercicio tengan un mayor conocimiento sobre la leche de cabra. La relación entre las principales opiniones recopiladas y la práctica de ejercicio se comparan en la Figura 4.

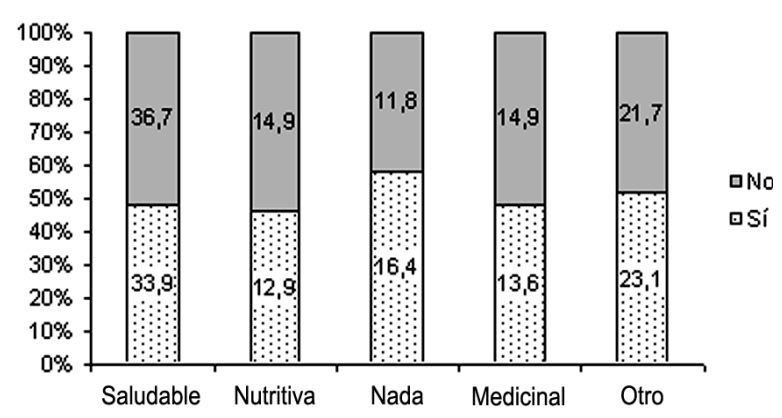

Figura 4. Distribución porcentual de las personas según actividad física dentro de las principales categorías de opinión manifestadas en la encuesta sobre la percepción y hábitos de consumo de leche y lácteos caprinos. San José, Costa Rica. 2007.

Si se observa la Figura 3, es notorio como un $14 \%$ de las personas encuestadas no conocen nada con respecto a la leche de cabra. Este último dato resulta interesante, pues según Vargas et al. (2007), entre jóvenes universitarios costarricenses entre los 17 y 26 años, el desconocimiento hacia la leche caprina llega a un nivel del $40 \%$. Una prueba de chi cuadrado encontró que existió relación entre el conocimiento sobre la leche de cabra según grupo de edad (X2=61,040; $<<0,0001)$. Los adultos jóvenes y los adolescentes fueron los dos grupos que más admitieron no tener ningún conocimiento sobre la leche de cabra (Figura 5).

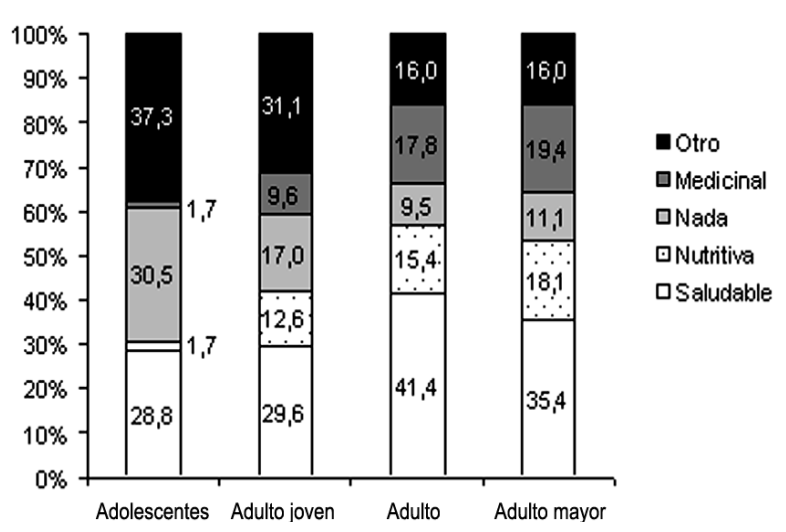

Figura 5. Distribución porcentual de las personas según edad dentro de las principales categorías de opinión manifestadas en la encuesta sobre la percepción y hábitos de consumo de leche y lácteos caprinos. San José, Costa Rica. 2007. 
El estudio de la Figura 5 permite evidenciar como la leche de cabra fue muy poco asociada con lo medicinal y lo nutricional por los adolescentes, e incluso los adultos jóvenes; esto si se contrastan los datos con lo expresado por los adultos y adultos mayores quienes la asocian más con estas características. Al parecer una menor curiosidad por alimentos saludables asociada con el vigor de las primeras edades podría estarse evidenciando en este trabajo para el caso particular de la leche de cabra, haciendo eco de los hallazgos de Vargas et al. (2007) en este mismo sentido. Más investigación de los hábitos y motivaciones del consumo de alimentos lácteos saludables es recomendable para este segmento poblacional, el cual suele ser un grupo poblacional en riesgo social en regiones de Latinoamérica y el Caribe (Maddaleno et al. 2003).

Las percepciones con respecto a la leche caprina también presentan una relación con el género $\left(\mathrm{X}^{2}=11,096 ; \mathrm{p}=0,0255\right)$ : fue mayor las proporción de mujeres que perciben la leche de cabra como "saludable" y "medicinal".

La distribución geográfica también marca algunos contrastes en cuanto a la percepción del producto. Un análisis evidenció que existieron relaciones entre las percepciones que tuvieron los encuestados sobre la leche de cabra según provincia $\left(\mathrm{X}^{2}=34,319 ; \mathrm{p}=0,0792\right)$. En la provincia de San José los entrevistados asociaron mayoritariamente la leche caprina con el concepto de "saludable" (42,2\% de los encuestados en la provincia), mientras que en la provincia de Limón la consideraron en menor medida como "nutritiva" $(4,1 \%$ de las personas). En Guanacaste $(14,7 \%)$ y Puntarenas $(10,4 \%)$, fue asociada estadísticamente en menor medida con propiedades medicinales.
Los detalles porcentuales de las percepciones según provincia se detallan en el Cuadro 3.

Al evaluar la variable consumo, un categórico 93,7\% de los encuestados (474 personas) mencionaron no tomar nunca leche de cabra. Esto es concordante con el hecho de que son los lácteos bovinos los que predominan en el mercado de Costa Rica (Corrales y Chacón 2005, Rojas et al. 2007). La población manifiestó opiniones relacionadas con las propiedades nutracéuticas de la leche de cabra, pero esto no se traduce en un consumo apreciable de la misma en ninguno de los grupos de edad o de los géneros, independientemente de la localización geográfica. Otro aspecto relevante es el que, aunque muchas personas tienen una opinión sobre la leche de cabra, la misma no se basa en una experiencia de consumo directa, planteándose la interrogante de si muchas de estas percepciones se forman mayoritariamente debido a tradiciones y a otras concepciones culturales.

Las razones por las cuales los participantes mencionaron que no toman leche caprina son la dificultad que implica conseguirla $(31,2 \%)$, y el "desagrado" que genera la misma $(31,2 \%)$. El desconocimiento hacia la leche, derivado de no haber sido ésta probada nunca, o por no acostumbrarse su uso en el círculo familiar figura en un tercer lugar $(14,6 \%)$. Entre otras razones misceláneas de no consumo se incluyen: "ninguna razón en especial" $(4,9 \%)$, "costumbre hacia la leche de vaca" $(2,7 \%)$, "desinterés" $(6,1 \%)$, "alto precio" $(2,5 \%)$ y "rechazo por los lácteos en general" (5,3\%). La Figura 6 muestra la relación gráfica de los resultados.

El sector caprino costarricense se caracteriza por estar conformado por pequeños y medianos productores,

Cuadro 3. Percepción porcentual de los encuestados sobre la leche de cabra según provincia de residencia. San José, Costa Rica. 2007.

\begin{tabular}{lccccccc}
\hline \multirow{2}{*}{ Percepción } & \multicolumn{6}{c}{ Percepción porcentual según encuestados por provincia (\%) } \\
\cline { 2 - 7 } & San José & Alajuela & Cartago & Limón & Heredia & Puntarenas & Guanacaste \\
\hline Saludable & 40,2 & 27,0 & 37,5 & 40,8 & 36,2 & 25,0 & 29,4 \\
Nutritiva & 10,3 & 21,3 & 10,7 & 4,1 & 10,6 & 20,8 & 26,5 \\
Nada & 13,0 & 11,2 & 19,6 & 12,2 & 14,9 & 22,9 & 11,8 \\
Medicinal & 10,9 & 14,6 & 12,5 & 24,5 & 21,3 & 10,4 & 14,7 \\
Otras & 25,5 & 25,8 & 19,6 & 18,4 & 17,0 & 20,8 & 17,6 \\
\hline
\end{tabular}




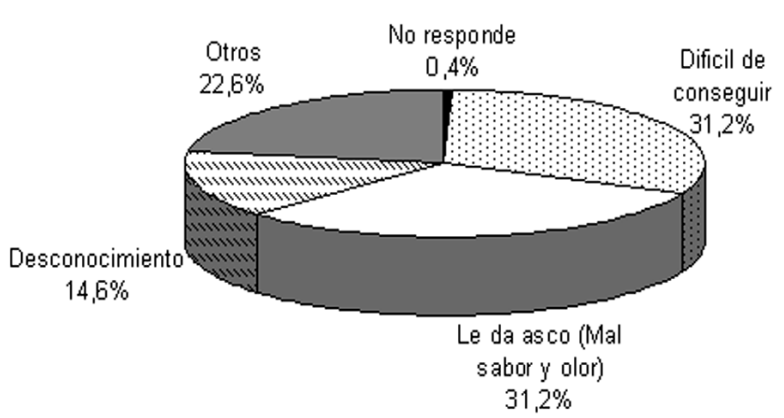

Figura 6. Distribución de las razones principales por las cuales los encuestados no toman leche de cabra. San José, Costa Rica. 2007.

quienes por lo general se encuentran distribuidos en todo el territorio costarricense, y que practican una venta limitada del producto a precios que por lo general son más altos que los de la leche de vaca (Tacsan 1987, Chacón 2004). Al parecer los problemas de distribución, precio y disponibilidad, derivados de este sistema agropecuario semiartesanal, influyen en la poca difusión (desconocimiento), y en la dificultad imperante para comprar el producto en el mercado. Más aún, puede presumirse que el manejo artesanal afectaría frecuentemente las características sensoriales de esta leche, lo cual puede en parte explicar los malos olores y sabores que se le atribuyen (Chacón 2005). El sabor suele ser una de las principales características con las que se evalúa la calidad de la leche en general (Santos et al. 2003, Drake 2004), aspecto al que se suma el aroma (Cais-Sokolinska et al. 2004). En el caso de la leche de cabra, el sabor es una consecuencia de muchos factores que se suponen están en un $80 \%$ relacionados con la alimentación del animal, en un 5\% con la oxidación de la fracción lipídica, en un 3\% con la higiene y en un $7 \%$ con causas misceláneas como el manejo (University of California 2007). La magnitud del impacto del manejo artesanal sobre las características sensoriales de la leche de cabra y su influencia sobre las opiniones del consumidor deben ser aún establecidas por estudios futuros. El bajo consumo de la leche caprina en el país parece estar vinculado con una pobre distribución y una consecuente desinformación, aspectos que en conjunto superan el rechazo debido a la náusea.

La evaluación estadística $\left(\mathrm{X}^{2}=10,890 ; \mathrm{p}=0,0278\right)$ encontró que la percepción de una sensación de desagrado asociado a la leche se relaciona con las mujeres
(35,5\% del total entrevistado). Al poseer la mujer un rol preponderante en la selección de los alimentos consumidos en el núcleo familiar costarricense y latinoamericano en general (Organización Panamericana de la Salud 2007), se perfila como un segmento meta en cuanto a la promoción de la leche caprina. El Cuadro 4 resume los datos de percepción según género.

Cuadro 4. Distribución de personas por género según las razones por las cuales no consumen leche de cabra. San José, Costa Rica. 2007.

\begin{tabular}{lcc}
\hline \multirow{2}{*}{$\begin{array}{c}\text { Razón de no } \\
\text { consumo }\end{array}$} & \multicolumn{2}{c}{ Género } \\
\cline { 2 - 3 } & Hombres (\%) & Mujeres (\%) \\
\hline No responde & 0,0 & 0,6 \\
Difícil de conseguir & 38,8 & 27,8 \\
Desagrado (náusea) & 21,8 & 35,5 \\
Desconocimiento & 16,3 & 13,8 \\
Otros & 23,1 & 22,3 \\
\hline
\end{tabular}

Se encontró una relación entre las razones por las cuales no se da un consumo de leche caprina con el grupo de edad $\left(\mathrm{X}^{2}=21,243 ; \mathrm{p}=0,0469\right)$. Los grupos de adulto y adulto mayor son quienes mencionaron en mayor porcentaje que la razón por la cual no toman leche de cabra es la poca disponibilidad, mientras que en el segmento de adultos jóvenes impera el desconocimiento, y en el de los adultos el asco (mal olor y sabor).

Los detalles porcentuales de las percepciones según grupo de edad se detallan en la Figura 7.

De los encuestados, únicamente 32 personas dijeron tomar leche de cabra (6,31\% del total), de los cuales es en San José $(37,5 \%)$ y Cartago $(21,8 \%)$ donde habitan mayoritariamente.

Un $34,4 \%$ de los consumidores de leche caprina fueron adultos mayores y un $43,75 \%$ adultos, lo cual podía asociarse con el hecho, antes determinado en este trabajo, que es precisamente éste el segmento donde hay un mayor interés y conocimiento por este alimento. Un 53\% hacen ejercicio contra un $47 \%$ que no lo hace, no siendo posible especular una posible relación del ejercicio con el consumo. 


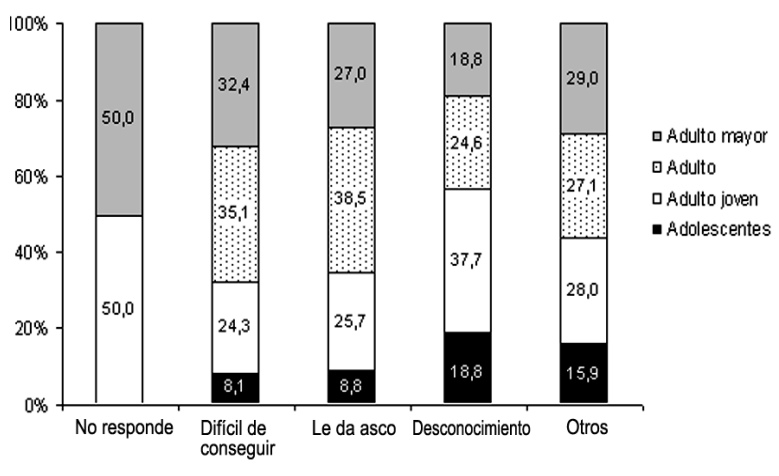

Figura 7. Distribución porcentual de las razones principales por las cuales los encuestados no toman leche de cabra, según grupo de edad. San José, Costa Rica. 2007.

Sobre el lugar de compra, un $68,75 \%$ de los encuestados que toman leche caprina afirmaron que la consumen, ya sea porque ellos mismos la producen, o porque la compran directamente a algún productor. Sólo el 31,25\% restante afirma adquirirla en algún tipo de comercio, principalmente pequeños detallistas. Este aspecto podría relacionarse con el manejo artesnal y con la poca disponibilidad de este producto en los comercios mayoristas, lo que impide su difusión y promoción.

Un 75\% de los consumidores habituales señaló que la principal motivación que tienen para consumir la leche caprina es el mejoramiento y la preservación de su salud. Únicamente un $18,75 \%$ señaló que lo hace por que le gusta el sabor del producto. La frecuencia de consumo resultó ser muy diversa, variando principalmente entre todos los días $(21,8 \%)$; una o más veces a la semana $(53,1 \%)$ y una vez al mes $(15,6 \%)$. Es interesante notar que entre estos consumidores, donde si existe disponibilidad de leche de cabra debido al autoabastecimiento o a la cercanía con un productor, la frecuencia de consumo es digna de tomar en consideración.

Con respecto al consumo de derivados de la leche de cabra, de los 507 encuestados afirmaron ingerirlos 57 individuos $(11,24 \%)$. De éstos, 36,8\% viven en San José, $21 \%$ viven en Alajuela y 17,5\% en Cartago. De las personas que consumen derivados, únicamente $11(19,3 \%)$ consumen también la leche de cabra. El consumo de estos derivados está constituido principalmente por el queso (80\%), el yogurt (10\%) y otros productos varios como dulces, fermentados, etc. A partir del consumo de lácteos, se puede inferir que la comercialización de leche fresca de cabra no es la única opción comercial de posible interés en el mercado nacional. Los derivados lácteos caprinos son adquiridos por los consumidores principalmente en pequeños negocios detallistas $(38,6 \%)$ o son comprados directamente al productor $(36,8 \%)$. Se destaca en los resultados una vez más el aspecto de disponibilidad y de procesamiento artesanal antes discutido. La frecuencia de consumo fue, al igual que en el caso de la leche fluida, muy variable, destacándose ingestas de una o dos veces por semana $(26,3 \%)$, una vez quincenal $(15,8 \%)$, o de una vez al mes $(24,6 \%)$ como las más usuales.

\section{CONCLUSIÓN}

La población estudiada se caracterizó por presentar una tendencia más marcada hacia el ejercicio en el sexo masculino, sin que esta tendencia esté supeditada significativamente a la edad de las personas. El consumo de leche de cabra, a pesar de ser considerado como saludable, no guardó ninguna relación con la práctica de algún ejercicio físico.

Las principales percepciones hacia la leche de cabra entre las personas evaluadas fueron el atribuirle a la misma un carácter de "saludable" (36\%), "nutritivo" (14\%) y "medicinal" (15\%). La concepción de la leche de cabra como un alimento nutracéutico se asoció con las personas del sexo femenino. La provincia de San José se mostró como aquella en que el carácter saludable de la leche caprina es más mencionado por los participantes $(42,2 \%)$, mientras que lo es menos en la provincia de Limón $(4,1 \%)$. Los grupos de edad más jóvenes fueron aquellos que se relacionaron con un mayor desconocimiento general hacia la leche de cabra.

Un 93,7\% de los entrevistados a nivel nacional no consume nunca leche de cabra. Es interesante notar como los conceptos positivos imperantes sobre la leche de cabra y sus propiedades no necesariamente se traducen en un indicador de alto consumo. Entre las principales razones argumentadas para no consumir este producto, se destacaron aspectos tales como la escasa disponibilidad especialmente enunciada por los 
grupos de mayor edad $(31,2 \%)$, la sensación de náusea especialmente asociada con las mujeres $(31,2 \%)$, y el desconocimiento, relacionado con los grupos de menor edad $(14,6 \%)$.

El 6,31\% de los encuestados que sí consumen la leche de cabra entre una y dos veces por semana $(53,1 \%)$, son generalmente adultos y adultos mayores distribuidos principalmente en las provincias de San José y Cartago, dónde compran la leche directamente a los productores $(68,75 \%)$ y motivados por su salud $(75 \%)$.

En cuanto a los derivados, un $11,24 \%$ de los encuestados que son personas que habitan principalmente en San José, Alajuela y Cartago, los consumen, estando principalmente representados estos derivados por el queso (80\%) y el yogurt $(10 \%)$. Estos son principalmente adquiridos con una frecuencia variable por medio de la compra a pequeños detallistas $(38,6 \%)$ o directamente con el productor $(36,8 \%)$.

A pesar de ser la leche de cabra percibida como un alimento saludable, es necesario una mayor producción y mercadeo del producto; no solo para modificar la percepción sensorial que de la misma se tiene, si no también para incrementar su consumo. Más estudios en este sentido se hacen necesarios ante la escasa disponibilidad de información actualizada al respecto.

\section{LITERATURA CITADA}

Cais-Sokolinska, D; Wojtowski, J; Pikul, J; Dankow, R. 2004. Quality and durability of fermented goat milk permeate. Archviert, Tierzuchtung Dummerstorf 47(1): 108-113.

Carriquiry, MR. 2005. Las políticas lecheras y su impacto en el consumo de lácteos. Taller panamericano: estrategias para incrementar el consumo de lácteos como aporte a la salud (en línea). Punta del Éste. 25 de abril del 2005. Consultado 23 mar. 2005. Disponible en: http://www. infoleche.com/fepale/fepale/MLMS/Ponenciastaller/ M.Carriquiry-consumo-ppt[1].ppt

Chacón, A. 2004. Acidez y peso específico de la leche de cabra de un grupo de capricultores de la meseta central costarricense. Agronomía Mesoamericana 15(2): 179-183.
Chacón, A. 2005. Aspectos nutricionales de la leche de cabra y sus variaciones en el proceso agroindustrial. Revista Agronomía Mesoamericana 16(2): 239-252.

Chacón, Y; Moncada, J. 2005. Aplicación del modelo trans teórico en los padres y encargados legales de los niños que participan en las escuelas deportivas y recreativas de la Universidad de Costa Rica. Revista Electrónica Actualidades Investigativas en Educación 5(2): 1-7.

Corrales, J; Chacón, A. 2005. Estudio de opinión de consumidores sobre el queso fresco de cabra (Capra hircus) en Costa Rica. Revista Agronomía Tropical 35: 39-49.

Díaz, C. 2004. Caracterización de la agroindustria láctea en Turrialba. Costa Rica. Revista Agronomía Tropical 34: 27-39.

Drake, M. 2004. ADSA Foundation scholar award: Defining Dairy flavors. Journal of Dairy Science 87(1): 777784.

Instituto Nacional de Estadística y Censos. 2005. Población total proyectada en Costa Rica por sexo, según años calendario (Hipótesis recomendada. 2000-2050) (en línea). Consultado 6 abril. 2005. Disponible en: http:// www.inec.go.cr

Ivankovich, C; Aguilar, F; Figueroa, J. 1990. Hábitos y motivaciones del consumo de queso en Costa Rica. CITA, Universidad de Costa Rica. San José. 28 p.

Maddaleno, M; Morello, P; Infante, F. 2003. Salud y desarrollo de adolescentes y jóvenes en Latinoamérica y el Caribe: desafíos para la próxima década. Salud Pública de México 45(1):s132-s139.

Meza, N. 2002. Hábitos alimentarios de la población costarricense (en línea). Consultado 20 nov. 2007. Disponible en: http://www.inciensa.sa.cr/ contenido/publicaciones/memorias_pdf/Contenido/ 7\%20Habitos\%20aliment $\% 20 \mathrm{CR} \% 20$.PDF

Ministerio de Salud de Costa Rica. 2001. Encuesta Carmen (en línea). Consultado 19 noviembre. 2007. Disponible en: http://www.ministeriodesalud.go.cr/ encuestas/encuesta\%20carmen.pdf

Morón, C; Alonso, L; Crovetto, M. 2005. Cambios en la estructura del consumo de alimentos y nutrientes de 
América Latina 1979-1981 a 1999-2001. Organización de las Naciones Unidas para la Agricultura y la Alimentación (FAO), Oficina Regional de la FAO para América Latina y el Caribe. Santiago de Chile. 63 p.

O’Mahony, M. 1986. Sensory evaluation of food. Statistical methods and procedures. Marcel Dekker, New York. $488 \mathrm{p}$.

Organización Panamericana de la Salud. 2007. Género y seguridad alimentaria (en línea). Consultado 19 noviembre. 2007. Disponible en: http://www.paho. org/Spanish/AD/GE/foodsecuritysp.PDF

Orozco, A.1999. Investigación de mercados: concepto y práctica. Norma. Bogotá, Colombia. 300 p.

Rojas, WN; Chacón, A; Pineda, ML. 2007. Características del yogurt batido de fresa derivadas de diferentes proporciones de leche de vaca y cabra. Revista Agronomía Mesoamericana 18(2): 221-237.

Santos, MV; Ma, Y; Caplan, Z; Barbano, DM. 2003. Sensory threshold of off-flavors caused by proteolysis and lipolysis in milk. Journal of Dairy Science 86 (1): 1601-1607.

Schiffman, L; Lazar, L. 2001. Comportamiento del consumidor. 7 ed. Prentice Hall, D.F. México. 300 p.

Severi, C; Girona, A. 2004. Los adolescentes, ¿consumen lácteos en cantidad suficiente? Foro electrónico panamericano: la importancia de los lácteos en la salud de los adolescentes. FEPALE / OPS (en línea). 1 al 17 de septiembre de 2004. Consultado 24 mar. 2006.
Disponible en: http://www.infoleche.com/fepale /fepale/lechesalud/documentos/documento 1 foro salud adolescente y lácteos.doc

Severi, C; Girona, A. 2005. Caracterización del consumo de lácteos en América Latina. Taller panamericano: estrategias para incrementar el consumo de lácteos como aporte a la salud (en línea). Punta del Éste. 25 de abril del 2005. Consultado 23 marzo 2005. Disponible en: http://www.infoleche.com/fepale/fepale/MLMS/ Ponenciastaller/Severi-Caracterización del consumo 2[1].ppt

Tacsan, I. 1987. Fabricación de un queso de cabra tipo crottin adaptado al gusto costarricense. Tesis Licenciatura en Tecnología de Alimentos. Universidad de Costa Rica. San Pedro de Montes de Oca. 83 p.

University of California. 2007. Milk quality and flavor (en línea). Consultado 15 feb. 2007. Disponible http:// www.saanendoah.com/milkqf.html

University of Maryland. 1992. National goat handbook (en línea). Consultado 16 nov. 2004. Disponible http:// www.inform.umd.edu/EdRes/topic/AgrEnv/ndd/goat

Vargas, P; Pineda, ML; Chacón, A. 2007. Lácteos bovinos y percepción de la leche caprina entre estudiantes de la Universidad de Costa Rica. Revista Agronomía Mesoamericana 18(1): 27-36.

Vega, O. 2002. Desempeño de la ganadería de leche y de la industria de la transformación de productos lácteos en Costa Rica 1999-2001. SEPSA, Área de Estudios Económicos e Información. San José. 32 p. 